\title{
CONSUMO DE FITOESTRÓGENO LINHAÇA E NÍVEIS LIPÍDICOS NA MENOPAUSA: O QUE HÁ DE EVIDÊNCIA?
}

\author{
CONSUPTIOM PHYTOESTROGEN FLAXSEED ALTERS LIPID PROFILES OF MENOPAUSAL: \\ WHAT IS EVIDENCE?
}

\section{Thais de Rezende Bessa Guerraa, Gilson Teles Boaventura ${ }^{b}$}

atcrezende34@gmail.com, bgilsontb@gmail.com

Universidade Federal Fluminense - Niterói (RJ), Brasil

Data de recebimento do artigo: 17/08/2015

Data de aceite do artigo: 04/04/2016

\section{RESUMO}

Introdução: A linhaça é um fitoestrógeno com ômega 3, lignanas e fibras que atuam de forma benéfica, promovendo ação hipolipidêmica. No período da menopausa, as mulheres estáo mais susceptíveis a alteraçóes no metabolismo lipídico, devido à privação estrogênica, e desenvolvem perfil lipídico altamente favorável à aterogênese. Na prática clínica, o óleo de linhaça e a linhaça in natura vêm sendo amplamente consumidos. Objetivo: Revisar as evidências na literatura sobre a influência do fitoestrógeno linhaça nas suas diferentes formas de ingestão in natura e óleo sobre o perfil lipídico, com ênfase em mulheres na menopausa e pós-menopausa. Métodos: Foram analisados 11 estudos que utilizaram como critérios: ensaios clínicos controlados, randomizados, crossover, internacionais e publicados entre 1997 e 2015 . Esta revisão utilizou dados de 360 mulheres na menopausa e pós-menopausa, com idade entre 41 e 73 anos que consumiram esse fitoestrógeno. Conclusáo: Os estudos apresentam curto período de consumo desse fitoestrógeno e a quantidade varia entre 20 a $40 \mathrm{mg} / \mathrm{dia}$, as evidências sugerem que o consumo de linhaça in natura influencia positivamente na redução dos níveis plasmáticos de colesterol total e LDL quando comparado ao consumo de óleo de linhaça.

Palavras-chave: Fitoestrógeno; linhaça; lipídio; mulheres; menopausa; evidência.

\section{ABSTRACT}

Introduction: Flaxseed is a beneficial phytoestrogen that has omega 3, lignans, and fiber that promotes hypolipidemic action. In the period of menopause women are more susceptible to changes in lipid metabolism, due to estrogen deprivation, and develop a lipid profile highly favorable to atherogenesis. In clinical practice the flaxseed oil and flaxseed in natura have been widely consumed. Objective: To review the literature evidence on the influence of phytoestrogen flaxseed in its different forms of intake in natura and oil on the lipid profile, with emphasis on menopausal and postmenopausal women. Methods: We analyzed 11 studies using as criteria: controlled clinical trials, randomized, crossover, international, and published between 1997 and 2015. This review used data from 360 menopausal and postmenopausal women, aged between 41 and 73 years who consumed this phytoestrogen. Conclusion: Studies have demonstrated short periods of consumption of this phytoestrogen and quantity ranging from 20 to $40 \mathrm{mg} / \mathrm{day}$, evidence suggests that the consumption of flaxseed in natura have a positive influence on reduction of plasma levels of total and LDL cholesterol as compared to consumption of linseed oil.

Keywords: Phytoestrogen; flaxseed; lipid; women; menopausal; evidence. 


\section{Introdução}

Os indicadores fornecidos pelo Ministério da Saúde mostram que as doenças cardiovasculares representam a primeira causa de morte no Brasil com 32,3\%, também ocorrendo nos EUA, onde $42 \%$ da mortalidade em adultos são causadas por doença arterial coronariana, tendo as mulheres os maiores índices ${ }^{1,2}$. Essa incidência aumenta substancialmente no começo da menopausa devido à perda da proteção do estrogênio. A redução desse hormônio favorece o surgimento da obesidade central, caracterizando um aumento da gordura visceral nessa fase fisiológica ${ }^{3}$, o que facilita o desenvolvimento das alteraçóes metabólicas, aumentando o risco cardiovascular, entre outras complicações, como as dislipidemias ${ }^{4}$.

As dislipidemias são caracterizadas por modificações no metabolismo lipídico que desencadeiam alteraçóes nas concentraçóes do colesterol total, triglicerídeos e lipoproteínas plasmáticas, podendo ocorrer de forma isolada ou combinada ${ }^{5}$. Essas modificaçóes favorecem o desenvolvimento de doenças crônicas como diabetes mellitus e doenças cardiovasculares ${ }^{6}$.

Dados epidemiológicos e experimentais demonstram uma associaçáa entre dieta e risco de desenvolver hipercolesterolemia, hipertrigliceridemia e ainda doenças cardiovasculares ${ }^{7,8}$. Destacam certos componentes dietéticos que têm função preventiva, como os alimentos funcionais ${ }^{9}$. Dessa forma, inúmeros estudos acerca do consumo crescente de alimentos considerados funcionais têm despertado interesse científico, como é o caso do consumo da semente de linhaça ${ }^{10}$.

O interesse no consumo desse fitoestrógeno se justifica pelos benefícios à saúde, devido às suas características quimioprotetoras ${ }^{6}$, cardioprotetoras $^{10}$, anticarcinogênicas, antioxidantes ${ }^{11}$ e por se comportar como um hormônio agonista ou antagonista, semelhante à molécula de estrogênio ${ }^{12}$.

Considerando a relevância do tema, este estudo tem como objetivo investigar as evidências na literatura sobre os efeitos do consumo das diversas formas de ingestão (in natura e óleo) da semente de linhaça na dieta de mulheres em período de menopausa e pós-menopausa e elucidar algumas questóes relacionadas ao consumo dessa semente por um período de tempo e sua ação nos indicadores cardiovasculares.

\section{Métodos}

Realizou-se uma revisão da literatura, nas bases de dados Medline (National Library of Medicine), LILACS, PubMed e Science Direct e dos comitês nacionais e internacionais de saúde, dos artigos publicados no período compreendido entre 1997 e 2015 . A busca eletrônica foi efetuada em abril de 2015, utilizando as seguintes palavras-chave: "flaxseed", "cholesterol", "triglicérides", "women menopausal" e "posmenopausal".

Os estudos selecionados foram de maiores evidências científicas, contemplando os ensaios clínicos controlados, randomizados e crossover.

Os critérios de inclusão foram: mulheres no período de menopausa e pós-menopausa, formas de consumo da semente de linhaça, dentre elas farinha, in natura, óleo de linhaça e alimentos suplementados, como muffins, farelos e margarina com óleo de linho.

A coleta de dados ocorreu da seguinte forma (Figura 1):

Figura 1: Etapas da coleta de dados.
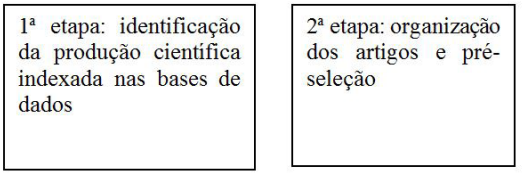

$3^{\text {a }}$ etapa: seleção dos artigos por meio de leitura dos resumos, utilizando critérios de inclusão e exclusão

- $\quad 1^{a}$ etapa: Identificação da produção científica indexada nas bases de dados pelos descritores citados, sendo encontrados 60 artigos, sendo 30 na base PubMed e 30 nas bases Medline, LILACS e SciELO;

- $\quad 2^{a}$ etapa: os artigos foram organizados em tabelas, contendo título, autor, revista, volume, ano de publicação, e pré-selecionados a partir da leitura do resumo disponível;

- $\quad 3^{a}$ etapa: a seleçáo dos artigos ocorreu por meio de leitura dos resumos, utilizando como critérios de inclusão publicações em português e inglês. No entanto, foram excluídos artigos com crianças e adolescentes (37), estudos repetidos nas diferentes bases de dados (16), repetidos quando as palavras-chave foram variadas (4) e os que não foram encontrados na íntegra (22).

Ao final, foram selecionados 11 artigos oriundos de comitês nacionais e internacionais de saúde para esta revisão.

A partir da análise, os dados obtidos foram organizados em tabelas, considerando o ano de publicação, o tipo de pesquisa e o enfoque na temática citados no corpo do artigo.

A partir da análise dos 11 artigos encontrados que apresentavam relevância com o tema, obteve-se uma média de 0,02 artigos produzidos no período de 1997 a 2015, o que revela uma necessidade de realizar mais estudos nessa temática, além de demonstrar a necessidade de incentivar e intensificar a produção científica nacional para aprofundamento na abordagem desse assunto. 
As publicações encontradas estão distribuídas em 11 periódicos diferentes, e é possível observar a predominância de produção nas revistas internacionais. Essa predominância talvez possa ser explicada pela maior destinaçáo de recursos financeiros para as pesquisas e pela existência de grandes centros de pós-graduaçáo, portanto, detentores de conhecimento.

Com a análise do conteúdo dos estudos, foi possível identificar as evidências sobre as formas de consumo da linhaça e os efeitos na menopausa e pós-menopausa.

\section{Resultados e discussão}

\section{Estudos experimentais}

As doenças cardiovasculares permanecem como a maior causa de mortalidade e morbidade entre as mulheres menopausadas ${ }^{11,12}$. Discussóes sobre a terapia de reposiçáo hormonal com estrógenos e progestógenos sintéticos são essenciais, visto o aumento constante no número de mulheres que consomem esse alimento nessa fase fisiológica ${ }^{13-15}$. O ômega 3, ácido graxo poli-insaturado, consiste em mais de $50 \%$ das gorduras totais presentes na semente de linhaça e age como protetor contra doenças cardiovasculares, diminuindo os teores séricos do colesterol total e triglicerídeos ${ }^{10}$.

Um estudo randomizado realizado no período de três semanas avaliou o consumo de muffin adicionado com farinha de linhaça desengordurada $50 \mathrm{mg} / \mathrm{dia}$ por um grupo (GFL) e muffin adicionado com farelo de trigo $20 \mathrm{mg} /$ dia por outro grupo (GLT) ${ }^{16}$, o GFL apresentou redução da lipoproteína de baixa densidade (LDL-c) e aumento nos níveis de triglicerídeos. O que corrobora os resultados apresentados em estudo realizado com mulheres que receberam de 5 a $10 \mathrm{~g} /$ dia de farinha de linhaça associado com fibra solúvel como suplemento ${ }^{17}$.

Em estudo realizado no período de três meses com mulheres na menopausa que consumiram $30 \mathrm{~g} / \mathrm{dia}$ de semente de linhaça e o segundo grupo que recebeu $30 \mathrm{~g} /$ dia farelo de trigo, não apresentaram diferenças estatísticas significativas no perfil lipídico ${ }^{18}$.

A busca pela suplementação da linhaça como terapia alternativa na fase da menopausa tem sido associada aos benefícios nos níveis lipídicos, prevenção cardíaca e melhora dos sintomas como fogachos ${ }^{19}$.

Em estudo ${ }^{19}$ randomizado, realizado no período de dois meses com dois grupos de mulheres na menopausa, um grupo recebeu $40 \mathrm{~g} /$ dia de semente de linhaça triturada (GL) e o outro $0,625 \mathrm{mg} / \mathrm{dia}$ de estrogênio isolado ou combinado com $100 \mathrm{mg} / \mathrm{dia}$ de progesterona (GTH: Grupo terapia hormonal), com prescrição para todos os grupos de dieta hipercolesterolêmica e prescrição para evitar o consumo de fitoestrógenos para o GTH. Os resultados apresentaram diminuição do LDL-c e aumento de lipoproteína de alta densidade (HDL-c) no GL, porém o GTH apresentou melhores e mais significativos resultados em relação ao perfil lipídico e marcadores relacionados à saúde cardiovascular. Ambos os tratamentos reduziram de forma similar os sintomas relacionados à menopausa e níveis séricos de glicose e insulina.

O estudo ${ }^{6}$ randomizado, crossover, dividiu dois grupos de mulheres pós-menopausadas que consumiram muffin de baixa gordura, com ou sem um complexo lignana, por seis semanas. Um grupo recebeu muffin com $42 \mathrm{~g} /$ dia de semente de linhaça, enquanto o outro recebeu muffin suplementado com secoisolariciresinol diglicosídeo (SDG) isolado (500 mg/dia). Os resultados encontrados mostraram que o muffin suplementado não alterou de forma significativa a concentração sérica de lipídio, enquanto o suplementado com semente de linhaça reduziu significativamente os triglicerídeos e LDL-c. Esses resultados estão associados à quantidade de fibras presente nessa oleaginosa que agem positivamente na diminuição do perfil lipídico ${ }^{20,21}$, auxiliando na prevenção de doenças cardiovasculares ${ }^{22}$.

Em estudo ${ }^{23}$ randomizado, duplo-cego, a população predominantemente afro-americana recebeu $3 \mathrm{~g} /$ dia de ácido alfalinolênico (ALA) em cápsulas de óleo de linhaça ou placebo com cápsulas de óleo de oliva. Os níveis plasmáticos de ácido eicosapentaenoico (EPA) e ácido docosahexaenoico (DHA) em 12 semanas no grupo do óleo de linhaça aumentaram 60 e $25 \%$, respectivamente, enquanto naqueles que consumiram óleo de oliva não houve alteração significativa. Esse estudo sugere a eficácia da conversão do ALA a EPA e DHA nessa população.

Em ensaio clínico ${ }^{24}$ randomizado, duplo-cego, no período de 26 semanas com consumo de 3 g/dia de ALA a partir de óleo de linhaça em cápsulas (GL) e placebo contendo cápsulas de azeite (GA), foram observados que no GL a concentração plasmática de colesterol total foi maior quando comparado ao GA, diminuindo os níveis de LDL-c, HDL-c, IDL-c e o tamanho de partícula lipídica. As concentraçôes de LDL-c apresentaram menos aterogênica no GL, e já LDL-c tendeu a ser maior no grupo GA. Foi considerado que ALA não alterou significativamente no GL o risco de doenças cardiovasculares, mas o tamanho da partícula e as concentraçóes das lipoproteínas plasmáticas.

No estudo ${ }^{25}$ sobre SDG extraído da linhaça com consumo de 300 e $600 \mathrm{mg} / \mathrm{dia}$ de pão e muffin, respectivamente, por oito semanas, os resultados foram significativos na redução de colesterol total, LDL-c, HDL-c e triglicerídeos. Sugere-se que a açáo fitoestrógena do SDG presente na semente de linhaça possa estar relacionada com a promoção da saúde cardiovascular ${ }^{25,26}$. 
Já no estudo ${ }^{27}$ randomizado realizado em quatro semanas com consumo de $20 \mathrm{~g} /$ dia de margarina obtida de óleo de linho reduziu a sensibilidade de HDL-c e aumentou a oxidação de LDL-c. O aumento acentuado na complacência arterial com ALA, presente no óleo de linho, reflete a melhora funcional na circulaçáo sistêmica. Sugere-se que a dieta com ácidos graxos n-3 confere uma nova abordagem para melhorar a função $\operatorname{arterial}^{27,28}$.

Nos resultados encontrados no estudo ${ }^{29}$ realizado a longo prazo, aproximadamente 12 meses, com dois grupos, no qual um recebeu pão com semente de linhaça na quantidade de $40 \mathrm{~g} /$ dia $(\mathrm{GL})$ e outro recebeu pão com farelo de trigo (GT), foram observadas influências positivas sobre o perfil lipídico no GL e não apresentaram diferenças significativas nos níveis séricos de colesterol total e triglicerídeos no GT.
No estudo randomizado, realizado no período de três meses com mulheres indígenas, hipertensas e na pós-menopausa, divididas em grupo controle (GC), grupo linhaça (GL) e grupo linhaça adicionado de aveia (GLA), os grupos GL e GLA consumiram $30 \mathrm{~g} /$ dia de semente in natura. Como resultado, o GL teve redução no perfil lipídico, e o GLA diminuição significativa dos triglicerídeos. Portanto, sugere-se uma melhor resposta plasmática no perfil lipídico quando associada às fibras de aveia adicionada à semente de linhaça.

Já estudo ${ }^{31}$ randomizado realizado em 12 semanas com consumo de cápsulas de óleo de linhaça (GL) e cápsulas de azeite (GA), sugere que não há diferenças estatísticas significativas no perfil lipídico entre GL e GA.

$\mathrm{Na}$ tabela a seguir, descrevem-se as formas de consumo da semente de linhaça (in natura e cápsula) e os efeitos no perfil lipídico no período da menopausa.

Tabela 1: Diferentes formas de consumo do fitoestrógeno linhaça e níveis lipídicos. Niterói (RJ), Brasil, 2015.

\begin{tabular}{|c|c|c|c|c|}
\hline Autor & Pacientes & $\begin{array}{l}\text { Período } \\
\text { do consumo }\end{array}$ & Intervençáo & Resultados \\
\hline Patade et al..$^{30}$ & 42 & 12 semanas & $\begin{array}{l}30 \mathrm{~g} / \mathrm{dia} \text { linhaça } \\
\quad \text { in natura }\end{array}$ & $\begin{array}{l}\text { Diminuiu o colesterol total (7\%), LDL (10\%) e au- } \\
\text { mentou os triglicerídeos (3\%) }\end{array}$ \\
\hline Lemay et al. ${ }^{19}$ & 25 & 16 semanas & $\begin{array}{l}40 \mathrm{~g} / \mathrm{dia} \text { linhaça } \\
\text { in natura }\end{array}$ & Diminuiu em 1,6\% de colesterol \\
\hline Lucas et al. ${ }^{5}$ & 36 & 12 semanas & $\begin{array}{l}40 \mathrm{~g} / \text { dia linhaça } \\
\text { in natura }\end{array}$ & $\begin{array}{c}\text { Diminuiu o colesterol total, não HDL, LDL e } \\
\text { triglicerídeos }\end{array}$ \\
\hline Jenkins et al. ${ }^{16}$ & 22 & 3 semanas & $\begin{array}{l}50 \mathrm{~g} / \text { dia linhaça } \\
\text { in natura }\end{array}$ & $\begin{array}{c}\text { Diminuiu o colesterol total, LDL, HDL, aumentou } \\
\text { triglicerídeos }\end{array}$ \\
\hline Hallund et al. ${ }^{6}$ & 22 & 6 semanas & $\begin{array}{l}500 \mathrm{mg} / \mathrm{dia} \text { SDG } \\
\text { isolado }\end{array}$ & $\begin{array}{l}\text { Não diminuiu o colesterol para o grupo que consumiu } \\
\text { SDG isolado; para o grupo que consumiu a semente de } \\
\text { linhaça houve diminuiçáo significativa do colesterol }\end{array}$ \\
\hline Zang et al. ${ }^{24}$ & 20 & 6 a 8 semanas & $\begin{array}{l}\text { Grupo } 300 \text { e grupo } \\
600 \mathrm{mg} / \mathrm{dia} \text { SDG isolado }\end{array}$ & $\begin{array}{l}\text { Diminuiu o colesterol total, HDL-c, LDL e } \\
\text { triglicerídeos }\end{array}$ \\
\hline Paul et al. ${ }^{27}$ & 15 & 4 semanas & $\begin{array}{l}20 \text { g de margarina } \\
(3 \text { g de ALA })\end{array}$ & $\begin{array}{l}\text { Colesterol LDL náo foi significativamente diferente } \\
\text { do grupo controle }\end{array}$ \\
\hline Harper et al. ${ }^{28}$ & 31 & 12 semanas & $\begin{array}{l}5,2 \text { g óleo de linhaça } \\
\text { (3 g de ALA) }\end{array}$ & $\begin{array}{c}\text { Aumentou } 70 \% \text { na concentração ALA, } \\
\text { Diminuiu 15\% de colesterol }\end{array}$ \\
\hline Harper et $\mathrm{al}^{23}$ & 49 & 26 semanas & $\begin{array}{l}5,2 \mathrm{~g} \text { de óleo de linhaça } \\
\text { (3 g de ALA) }\end{array}$ & Não houve alteração do HDL, LDL e triglicerídeos \\
\hline Dodin et al. ${ }^{29}$ & 98 & 12 meses & $\begin{array}{l}40 \mathrm{~g} / \text { dia linhaça } \\
\text { in natura }\end{array}$ & Diminuiu o colesterol total \\
\hline Kaul et al. ${ }^{31}$ & 86 & 12 semanas & $\begin{array}{l}2 \mathrm{~g} / \text { dia de óleo de linhaça } \\
\text { e óleo de azeite }\end{array}$ & $\begin{array}{l}\text { Não houve alteraçăo do CT, HDL, LDL e } \\
\text { triglicerídeos }\end{array}$ \\
\hline
\end{tabular}

Essa oleaginosa contém $41 \%$ de lipídios, $28 \%$ de fibras, $21 \%$ de proteínas, $6 \%$ de carboidratos e $4 \%$ de restos de sementes. Contém mistura de ácidos graxos poli-insaturados, sendo $57 \%$ de ácido alfalinolênico e $16 \%$ de ácido linoleico, considerado boa fonte de lipídios, ômega 3 e ômega 6, respectivamente ${ }^{32}$. Também são considerados ácidos graxos essenciais, pois não podem ser biossintetizados pelo homem, devendo ser ingeridos na alimentação ${ }^{33}$.
O ômega 3 atua na prevenção da hipertensão arterial, diminuindo os riscos de infarto agudo do miocárdio, cânceres, artrite reumatoide e prevenção de osteoporose, esclerose múltipla e insuficiência renal ${ }^{34-36}$.

Estudos clínicos com dietas ricas em ácidos graxos poli-insaturados relatam os benefícios na prevenção de doenças cardíacas $^{34}$, prevenção de arritmias, geração de prostanoides e leucotrienos com ações inflamatórias e inibição de síntese de citocinas por serem precursores dos ecosainoides, como 
as tromboxanas, prostaglandinas (substâncias que promovem a vasodilatação) e prostaciclinas que atuam reduzindo a agregação plaquetária e aumento da síntese de $\mathrm{HDL}^{21,34}$.

$\mathrm{O}$ aumento no consumo da semente de linhaça em mulheres na menopausa tem sido associado às suas propriedades fitoestrogênicas como melhora dos sintomas clínicos, fogachos e melhora no perfil aterogênico, pois essa semente possui um componente com estrutura química semelhante à molécula do SDG, a fonte mais rica deste componente está presente em média $52,7 \mathrm{mg}$ de lignana/100 $\mathrm{g}^{26,37}$. Esse composto se liga aos receptores de estrógeno (RE) alfa e beta em órgãos-alvo e vem sendo utilizado na terapia de reposiçáo hormonal alternativa em substituição à ação de esteroides ovarianos, quando a síntese apresenta-se gravemente reduzida no período da menopausa ${ }^{37,38}$. Estudos demonstram que a suplementação com a semente de linhaça aumenta os níveis dos hormônios sexuais nessa fase fisiológica e estão associados a alteraçóes no perfil lipídico aterogênico ${ }^{5}$ à diminuição dos riscos não só de doenças cardiovasculares ${ }^{37}$, mas também de câncer de mama ${ }^{38}$, além de auxiliar na prevenção dos sintomas característicos da menopausa ${ }^{39,40}$.

Diante do exposto, observa-se que o consumo dessa semente na forma in natura apresenta melhora no controle do perfil lipídico quando comparado ao consumo com cápsula de linhaça. Entende-se que efeito cardioprotetor está associado ao ômega 3, lignanas e fibras ${ }^{36,37}$.

\section{Conclusão}

As mulheres na menopausa e pós-menopausa estáo mais susceptíveis a alterações no metabolismo lipídico devido à privação estrogênica. Considerando as evidências na literatura sobre o consumo da semente de linhaça e suas formas de ingestão, como óleo ou in natura, sobre o perfil lipídico nessa população e fase fisiológica, observa-se que o consumo desse fitoestrógeno na forma in natura apresenta melhor eficácia sobre o perfil lipídico e consequente proteção cardiovacular quando comparado ao consumo com cápsula de linhaça. Sugere-se que o efeito cardioprotetor está associado ao ômega 3, lignanas e fibras presentes na forma de consumo in natura.

\section{Referências}

1. Brasil. Ministério da Saúde. Indicadores e Dados Básicos/ FUNASA CENEPi. Sistema de Informaçóes sobre mortalidade. [Internet]. 2001. [acesso em 12 jul 2016]. Disponível em: http://tabnet.datasus.gov.br

2. Organização Mundial da Saúde. Investigaciones sobre la menopausia en los años noventa. Ginebra; 1996. (Serie de Informes Técnicos, n. 866).
3. Guyton AC. Fisiologia Humana. 6a ed. Rio de Janeiro: Guanabara Koogan; 1998.

4. World Health Organization. Population division. Department of economic and social affairs. Ageing. Disponível em: www.popin.org/pop1998/8htm

5. Lucas EA, Lightfoot SA, Hammond LJ, Devareddy L, Khalil DA, Daggy BP, et al. Flaxseed reduced plasma cholesterol and atherosclerotic lesion formation in ovarectoized golden Syrian hamsters. Atherosclerosis. 2004;173(2):223-9.

6. Hallund J, Ravn-Haren G, Bugel S, Tholstrup T, Tetens I. Lignan complex isolated from flaxseed does not affect plasma lipid concentrations or antioxidant capacity in health postmenopausal women. J Nutr. 2006;136(1):112-6.

7. Kannel W. Menopause and risk of cardiovascular disease: the Framingham study. Ann Intern Med. 1976;85:447-52.

8. Prasad K. Effect of chronic administration of lignan complex isolated from flaxseed on the hemopoietic system. Molecular and cellular biochemistry. 2005;270(1-2):139-145.

9. Begum NA, Nicole C, Milla I, Lapierre N, Fukushima K. Dietary lignans precursor of mammalian lignans in rats. J Nutr. 2003;134(1):120-7.

10. Ward WE, Chen J, Thompson LU. Exposure to flaxseed or its purified lignan during suckling only or continuously does not alter reproductive indices in male female off spring. J Toxicol Environ Health. 2001;64(7):567-77.

11. 11. Xavier HT, Izar MXC, Faria Neto JR., Assad MH, Rocha VZ, Sposito AC, et al. V Diretriz Brasileira de dislipidemia e aterosclerose. Arq Bras Cardiol. 2013;101(4, Supl 1).

12. Guerra TRB, Boaventura GT, Vellarde LG. Phytoestrongens the modulador selective receptor estrogen? Rev Ciên Méd Biol. 2011;10(1):67-70.

13. Branca F, Lorenzetti S. Health effects of phytoestrogens. Forum Nutr. 2005;57:100-11.

14. Botsis D, Christodoulakos G, Papagianni V, Lambrinoudaki I, Aravantinos L, Makrakis E, et al. The effect of raloxifene and tibolone on the endometrium as assessed by bleeding episodes: tranvaginal scan and endometrial biopsy. Climateric. 2005;7(3):261-6.

15. Mikkola TS, Clarkson TB. Estrogen replacement therapy, atherosclerosis and vascular function. Cardiovas Res. 2002;53(3):605-19.

16. Jenkins DJA, Kendall CWC, Vidgen E, Agarwal S, Rao AV, Rosenberg RS, et al. Health aspects of partially defatted flaxseed: effects on serum lipids, oxidative measures and ex vivo androgen and progestin activities: a controlled crossover trial. Am J Clin Nutr. 1999;69:395-402.

17. Hutchins AM, Slavin JL. Effects of flaxseed on sex hormones metabolism. In: Thompson LU, Cunnane SC. Flaxseed in Human Nutrition. $2^{\mathrm{a}}$ ed. Champaign: AOCS; 2003. p. 126-49.

18. Lucas EA, Wild RD, Hammond LJ, Khalil DA, Juma S, Daggy B, et al. Flaxseed improves lipid profile without altering biomarkers of bone metabolism in postmenopausal women. J Clin Endocrinol Metab. 2002;87(4):1527-32. 
19. Lemay A, Dodin S, Kadri N, Jacques H, Forest JC. Flaxseed dietary supplement versus hormone replacement therapy in hypercholesterolemic menopausal women. Obstet Gynecol. 2002;100(3):495-504.

20. Belda MCR, Pourchet-Campos MA. Ácidos graxos essenciais em nutrição: uma visão atualizada. Ciên Tecnol Aliment. 1991;11(1):5-35.

21. Turatti JM, Gomes RAR, Athié I. Lipídios: aspectos funcionais e novas tendências. Campinas, ITAL; 2002. p. 78.

22. Oliveira A, Filho MJ. Perfil nutricional e lipídico de mulheres na pós-menopausa com doença arterial coronariana. Arq Bras Cardiol. 2005;84(4):325-9.

23. Harper CR, Edwards MJ, Jacobson TA. Flaxseed oil supplementation does not affect plasma lipoprotein concentration or particle size in human subjects. J Nutr. 2006;136(11):2544-8.

24. Zang W, Xiaobing W, Yi L, Haimei T, Brent F, Mark W, et al. Dietary flaxseed lignan extract lowers plasma cholesterol and glucose concentrations in hypercholesterolaemic subjects. Br J Nutr. 2008;99:1301-9.

25. Leanne TB, Shilpa B, Jesse C, Stephen C, Cunnane JA, Berlin DJ, et al. Flaxseed and cardiovascular risk factors: results from a double blind, randomized, controlled clinical trial. J Am College Nutr. 2008;27(1):65-74. 2008.

26. Claupach R, Meirelles RMR, Julião MA, Kohler SG, Loureiro CC, Giarodoli PB, et al. Fitoestrogênicos: posicionamento do Departamento de Endocrinologia Feminina da Sociedade Brasileira de Endocrinologia e Metabologia (SBEM). Arq Bras Endocrinol Metab. 2002;46(6):679-95.

27. Paul J, Nestel SE, Pomeroy TS, Takeshi Y, Yu LL, Anthony $\mathrm{M}$, et al. Arterial compliance in obese subjects is improved with dietary plant n-3 fatty acid from flaxseed oil despite increased LDL oxidizability arterioscler. Thromb Vasc Biol. 1997; 17:1163-70.

28. Harper CR, Edwards MJ, Filipis AP, Jacobson TA. Flaxseed oil increases the plasma concentrations of cardioprotective (n-3) fatty acids in humans. J Nutr. 2006;36(1):83-7.

29. Dodin S, Lemay A, Jacques H, Légare F, Forest JC, Maassê B. The effects of flaxseed dietary supplement on lipid profile, bone mineral density, and symptoms in menopausal women: A randomized double-blind, wheat germ placebo-controlled clinical trial. J Clin Endocrinol Metab. 2005;90(3):1390-7.
30. Patade A, Devareddy L, Lucas EA, Korlagunta K, Daggy BP, Arjmandi BH. Flaxseed reduces total and LDL cholesterol concentrations in Native American postmenopausal women. J Women Health (Larchmt). 2008;17(3):355-66.

31. Kaul N, Kreml R, Austria JA, Richard MN, Edel AL, Dibrov E, et al. A comparison of fish oil, flaxseed oil and hempseed oil supplementation on selected parameters of cardiovascular health in healthy volunteers. J Am Coll Nutr. 2008;27(1):51-8.

32. Canada Western Flaxseed an of Yellow Flaxseed Samples. Canadian Grains Comission, Winninpeg, MB, 2001. Disponível em: www.grainsscanada.dc.ca

33. Cucanne SC, Ganguli S, Menard C, Liede AC, Hamadeh MJ, Chen ZY, et al. Hight alpha-linolenic acid flaxseed (linum usitatissimum): some nutrition properties in humans. Bras J Nutr. 1993;69:443-53.

34. Kuijsten A, Bas H, Bueno M, Jolanda M, Boer ILJAC, Arts FJ, et al. Plasma enterolignans are not associated with nonfatal myocardial infarction risk. Atherosclerosis. 2009;203:145-52.

35. Carreau C, Gilles F, Catherine B, Potier M. Enterodiol and enterolactone, two major diet-derived polyphenol metabolites have different impact on Er $\alpha$ transcriptional activation in human breast cancer cells. J Steroid Biochem Mol Biol. 2008;11:176-85.

36. Guerra TRB. The influence of flaxseed on the body weight of wistar ovarioctomized rats. Rev Atenção à Saúde. 2015;13(43):11-7.

37. Guerra TRB. Efeito do consumo da semente de linhaça em ratas castradas: indicadores hormonais e imuno-histoquímicos. Dissertação [Mestrado em Ciências Médicas] Universidade Federal Fluminense; 2011.

38. Hu C, Yuan YV, Kitts DD. Antioxidant activities of the flaxseed lignan secoisolariciresinol diglucoside, its aglycone ecoisolariciresinol and the mammalian lignans enteroldiol and enterolactone in vitro. Food Chem Tox. 2007;45:2219-27.

39. Arjmandi $\mathrm{BH}$. The role of phytoestrogens in the prevention and treatment of osteoporosis in ovarian hormone deficiency. J Am Coll Nutr. 2001;20(5):398S-402S.

40. The European Heart Network. Food, nutrition and cardiovascular disease prevention in the European region: Challenges for the Millennium. 2002. Disponível em: http://www.ehnheart.org/component/downloads/downloads/1386.

\section{Como citar este artigo:}

Guerra TRB, Boaventura GT. Consumo de fitoestrógeno linhaça e níveis lipídicos na menopausa: o que há de evidência?. Rev. Aten. Saúde. 2016;14(49):92-97. 